\title{
Síndrome del túnel del tarso
}

\section{Tarsal tunnel syndrome}

\section{Antonio Cisneros Fuentes*}

Citar como: Cisneros FA. Síndrome del túnel del tarso. An Med (Mex). 2021; 66 (1): 54-56. https://dx.doi.org/10.35366/99490

\begin{abstract}
RESUMEN
Los síndromes por compresión nerviosa constituyen un grupo de patologías que podemos diagnosticar con mayor frecuencia, es importante conocer su existencia y la anamnesis. Se presenta el caso de una paciente con plantalgia crónica de tipo neuropático quien debió pasar por distintos médicos, estudios y tratamientos sin resultados satisfactorios antes de poderse integrar un diagnóstico de síndrome del túnel del tarso y así lograr un tratamiento efectivo que le incorporara a sus actividades cotidianas. Se debe conocer sobre las neuropatías compresivas y su abordaje clínico para poder sospechar de su presencia ante una plantalgia.
\end{abstract}

Palabras clave: Síndrome del túnel tarsiano, plantalgia, neuropatía compresiva.

\begin{abstract}
Nerve compression syndromes constitute a group of pathologies that we can diagnose more frequently, it is important to know their existence and the anamnesis. We present the case of a patient with chronic plantalgia who had to go through different doctors, studies and treatments without satisfactory results before being able to integrate a diagnosis of tarsal tunnel syndrome and thus achieve an effective treatment that incorporated her into her daily activities. It is necessary to know about compressive neuropathies and their clinical approach to be able to suspect their presence in plantalgia.
\end{abstract}

Keywords: Tarsal tunnel syndrome, plantalgia, compressive neuropathy.

\section{INTRODUCCIÓN}

* Ortopedista/Cirujano de Pie y Tobillo. Médico adscrito al Instituto Nacional de Rehabilitación (INR LGII). Médico adscrito al Instituto Mexicano del Seguro Social (IMSS). Hospital Ángeles Pedregal, H. San Ángel Inn Patriotismo.

Recibido: 10/01/2021. Aceptado: 20/03/2021.

Correspondencia: Dr. Antonio Cisneros Fuentes Hospital Ángeles Pedregal

Camino a Santa Teresa Núm. 1055, Consultorio 608,

Col. Héroes de Padierna 10700,

Alcaldía Magdalena Contreras, CDMX

Tels: 55 5568-4081, 55 5568-2422 y 55 5652-6346

E-mail: acisnerosf@hotmail.com

Abreviaturas:

$\mathrm{RM}=$ Resonancia magnética.

$\mathrm{TP}=$ ibial posterior .

$\mathrm{FHL}=$ Flexor largo del hallux .

US $=$ Ultrasonido

FD = Flexor común de los dedos.
Los síndromes por compresión nerviosa constituyen un grupo de patologías que podemos diagnosticar con mayor frecuencia, es importante conocer su existencia y la anamnesis. ${ }^{1,2}$

Un síndrome de atrapamiento nervioso resulta de la compresión de una rama nerviosa por alguna estructura, teniendo etiologías y manifestaciones clínicas diversas. Pero en general el cuadro clínico se caracteriza por parestesias y dolor neuropático en trayectos nerviosos. ${ }^{2-4}$

\section{PRESENTACIÓN DEL CASO}

Se trata de paciente femenino quien a los 14 años (en 2016) inicia con dolor incipiente que progresa a moderada intensidad, localizado en región plan- 
tar medial y de predominio en lateral del medio pie derecho.

Su médico tratante (septiembre de 2016) solicita estudio de imagen resonancia magnética (RM), la cual reporta: tenosinovitis del tibial posterior (TP) y flexor largo del hallux (FHL) así como aumento de líquido presente en espacio intercuneano y metatarsal entre segundo y tercer radios; además de presencia de os trigonum. Decide entonces infiltración de esteroide en corredera retromaleolar del TP y FHL así como en segundo espacio intermetatarsal (Figura 1).

Sin mejoría del cuadro sintomático y seis meses después la paciente acude con un segundo especialista en 2017 quien decide realizar resección artroscópica del os trigonum.

La paciente se refiere sin cambios en su cuadro doloroso y a lo largo de más de tres años el dolor plantar medial y lateral, de carácter urente, se asocia a hipoestesia en la raíz de los dedos cuarto y quinto menores. Hasta que a los 18 años de edad se exacerba con hiperalgesia e hiperbaralgesia que impide la marcha normal por dolor intenso durante el apoyo plantar, por lo que acude con nosotros en septiembre de 2020.

A la exploración física, la paciente se desplaza con asistencia de muletas sin apoyo de MPD, dolor 7/10 de EVA localizado en región plantar medial y de predominio lateral, con hiperbaralgesia a la digitopresión local y en raíz de los dedos menores, dolor sutil a la pronosupinación y plantiflexión máxima. Dolor urente a la palpación retromaleolar medial que se irradia proximalmente. Signo de túnel en dicha región además de prueba de Silfverskiold son positivos. Al evaluar con la escala AOFAS para retropié arroja una puntuación de 24.

\section{Investigaciones o exámenes}

Presenta estudios de imagen (agosto de 2020), placas simples y ultrasonido (US) musculoesquelético, el cual reporta: sin evidencia de lesión demostrable.

Se solicita entonces nueva RM, de la cual se informa: tenosinovitis del TP, FHL y del flexor común de los dedos (FD) así como aumento de líquido pre-
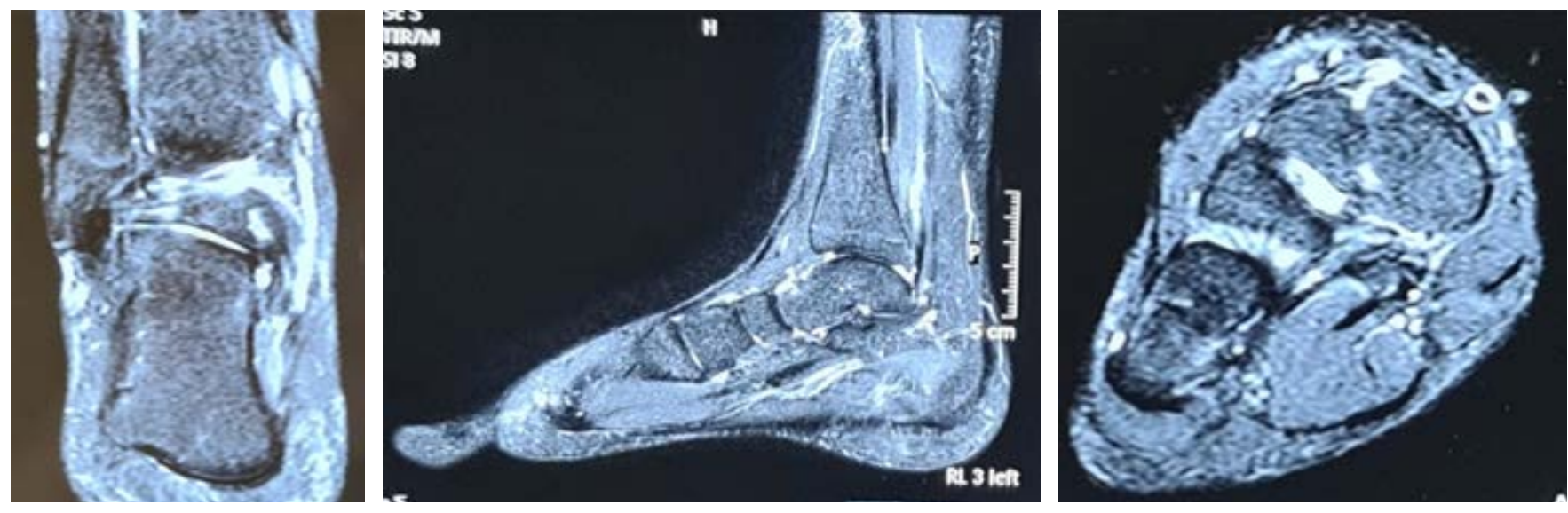

Figura 1: Resonancia magnética, septiembre de 2016.
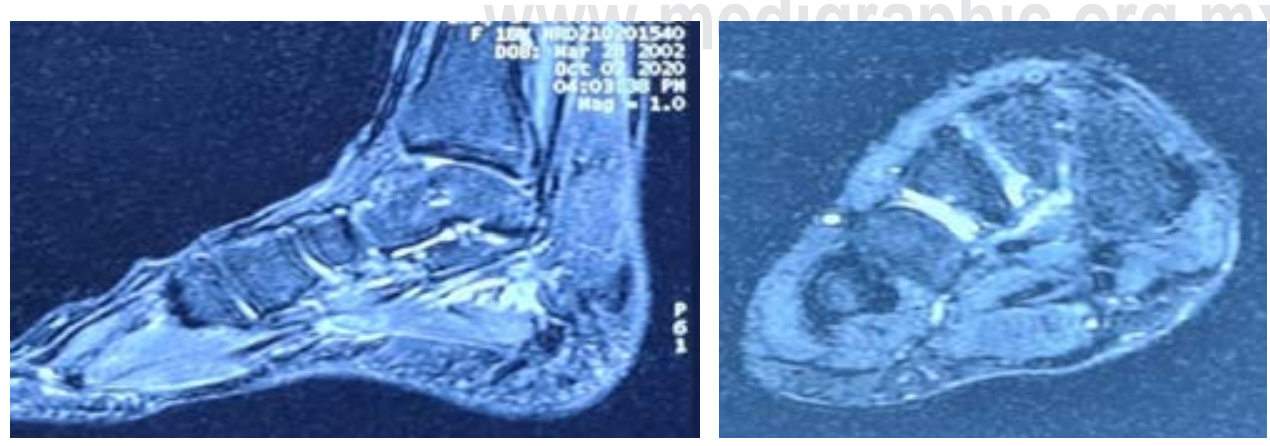

Figura 2:

Resonancia magnética, octubre de 2020. 


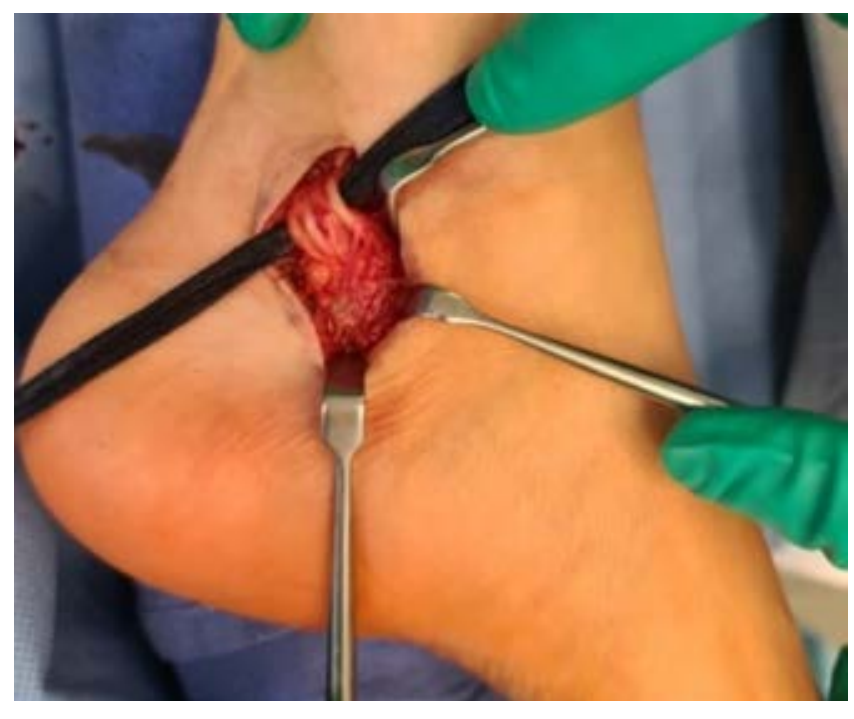

Figura 3: Liberación del paquete neurovascular.

sente en espacio entre segundo y tercer cuneiformes (Figura 2).

El estudio electromiográfico muestra: neuropatía compresiva del nervio tibial posterior en sus ramas, en un nivel un centímetro distal al maléolo medial (proximal del retináculo flexor), con datos de denervación para la rama plantar lateral, con datos de cronicidad.

\section{Tratamiento}

En octubre de 2020 se realizó una liberación abierta del túnel del tarso por sección del ligamento lacinado, con exploración y neurólisis de sus ramas plantar medial y lateral; además de un alargamiento fraccionado del tendón calcáneo por vía percutánea. En el mismo acto se aplicó en el perineuro una solución de betametasona con bupivacaína (Figura 3).

\section{RESULTADOS}

La evolución en el postoperatorio inmediato fue muy buena, a los tres días del procedimiento sin referir el dolor neuropático, y a los 15 días ya había apoyo de la extremidad, de manera que se retiraron puntos de sutura a los 19 días, en los que ya se realizaba marcha independiente con carga total. Con un puntaje de 96 aplicando la escala AOFAS para retropié. A las nueve semanas la paciente restablecida con AOFAS de 100 fue capaz de esquiar sobre nieve.

\section{DISCUSIÓN}

Un gran número de pacientes padece de plantalgia en algún momento de su vida, y la incidencia global de este síndrome se ha reportado entre 11 y $15 \%$ de la población en alguna etapa de su vida según estudios de varios autores. ${ }^{5}$ Así Yunoki reporta buenos resultados postquirúrgicos en una serie de seis pies intervenidos por liberación del túnel tarsal y recidivas en uno de ellos; por lo que recomienda considerar el empleo de medios preventivos de granulación y antiadhesivos posteriores a la descompresión quirúrgica. $^{6}$

\section{CONCLUSIÓN}

El diagnóstico depende de la exploración física con pruebas clínicas además de estudios de imagen como la RM y US, y estudios de electrodiagnóstico para llegar a un diagnóstico de certeza.

\section{REFERENCIAS}

1. Martín-Oliva X, Elgueta-Grillo J, Veliz-Ayta P, OroscoVillaseñor S, Elgueta-Grillo M, Viladot-Perice R. Variantes anatómicas del nervio calcáneo medial y nervio de Baxter en el túnel del tarso. Acta Ortop Mex. 2013; 27 (1): 38-42.

2. Guerrero OS, Coheña JM, Montaño JP, Perea RJ, Alfonso GN. Síndromes de atrapamiento nervioso en el pie: túnel tarsiano, túnel tarsiano anterior y atrapamiento del nervio de Baxter. Rev Esp Pod. 2015; 26: 134-138.

3. Cimino WR. Tarsal tunnel syndrome: review of the literature. Foot Ankle. 1990; 11 (1): 47-52.

4. Ahmad M, Tsang K, Mackenney PJ, Adedapo AO. Tarsal tunnel syndrome: a literature review. Foot Ankle Surg. 2012; 18 (3): 149-152.

5. Moroni S, Zwierzina M, Starke V, Moriggl B, Montesi F, Konschake M. Clinical-anatomic mapping of the tarsal tunnel with regard to Baxter's neuropathy in recalcitrant heel pain syndrome: part I. Surg Radiol Anat. 2019; 41 (1): 29-41.

6. Yunoki M. Analysis of surgical cases of tarsal tunnel syndrome in our department: case series and literature review. Asian J Neurosurg. 2020; 15 (1): 59-64. 